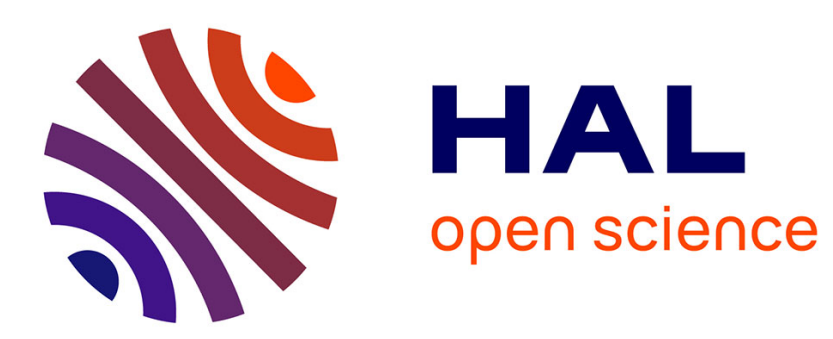

\title{
Experimental study of a supersonic jet-mixing layer interaction
}

\author{
Erwan Collin, Stéphane Barre, Jean-Paul Bonnet
}

\section{To cite this version:}

Erwan Collin, Stéphane Barre, Jean-Paul Bonnet. Experimental study of a supersonic jet-mixing layer interaction. Physics of Fluids, 2004, 16 (3), pp.765-778. 10.1063/1.1644574 . hal-00182572

\section{HAL Id: hal-00182572 \\ https://hal.science/hal-00182572}

Submitted on 5 Feb 2020

HAL is a multi-disciplinary open access archive for the deposit and dissemination of scientific research documents, whether they are published or not. The documents may come from teaching and research institutions in France or abroad, or from public or private research centers.
L'archive ouverte pluridisciplinaire HAL, est destinée au dépôt et à la diffusion de documents scientifiques de niveau recherche, publiés ou non, émanant des établissements d'enseignement et de recherche français ou étrangers, des laboratoires publics ou privés. 


\title{
Experimental study of a supersonic jet-mixing layer interaction
}

\author{
E. Collin ${ }^{\text {a) }}$ \\ LEA, BP 179, 86960 Futuroscope Cedex, France \\ S. Barre \\ LEGI, BP 53, 38041 Grenoble Cedex 9, France \\ J. P. Bonnet \\ LEA, BP 179, 86960 Futuroscope Cedex, France
}

(Received 14 May 2003; accepted 14 November 2003; published online 4 February 2004)

An experimental study is performed to analyze the interaction between a control jet (CJ) and a moderately supersonic main jet. Flow visualizations and laser Doppler velocimetry methods are used. A strong instability of the CJ has been identified. The dynamic of this instability corresponds to that of the local mixing layer. Two stability scenarios are proposed, one corresponding to the local Kelvin-Helmholtz instability of the main jet, the other linked to a local absolute instability of the interaction. The impact on the turbulent quantities is analyzed. It is shown that a strong modification of the Reynolds stress is manifest but that this extends only a small distance from the interaction.

(C) 2004 American Institute of Physics. [DOI: 10.1063/1.1644574]

\section{INTRODUCTION}

In many industrial applications, especially in ejectors and propulsive jets, most of the dynamical behavior of the system is strongly influenced by the mixing efficiency in a supersonic jet. This is particularly the case when enhanced mixing of hot propulsive jets is required, for example, to reduce the infrared signature of military aircrafts. Aircraft manufacturers are thus trying to develop low-cost, efficient mixing enhancement systems.

Such devices interact with the turbulent structures of the flow. It is not the purpose of this paper to deal in detail with the different instabilities of round jets. For this, let us recall from the major references on this topics (see, for instance, Bernal and Roshko ${ }^{1}$ and Crow and Champagne ${ }^{2}$ ). The principle instability is related to the column mode, i.e., the global mode of the jet, directly related to the Strouhal number based on the global characteristics of the jet:

$$
S t_{D}=\frac{f_{D} \cdot D}{U_{J}},
$$

where $f_{D}$ is the frequency of the jet column instability, $D$ the jet diameter, and $U_{J}$ the jet exit velocity. This Strouhal number characterizes the entire jet. In addition, it should be recalled that the round jet comprises an axisymetric mixing layer originating at the nozzle trailing edge. Before converging at the end of the potential core, with different spatial modes whose size increase in the downstream direction, and whose temporal modes also vary with axial position. For these instabilities, local characteristics such as the vorticity thickness $\delta_{\omega}(x)$ constitute the relevant parameters. Thus, a local Strouhal number can be introduced

\footnotetext{
a) Author to whom correspondence should be addressed. Telephone: (33) 5 $4936 \quad 60$ 33; fax: (33) $5 \quad 49 \quad 36 \quad 60$ 01; electronic mail: erwan.collin@lea.univ-poitiers.fr
}

$$
S t_{M L}=\frac{f_{M L} \cdot \delta_{\omega}}{U_{C}}
$$

where $f_{M L}$ is the local frequency of the Kelvin-Helmholtz instability, $\delta_{\omega}$ the local mixing layer vorticity thickness, and $U_{C}$ the convective velocity associated with the KelvinHelmholtz eddies.

Two kinds of mixing enhancement systems are generally distinguished: Passive and active.

Passive devices are defined as devices that do not need energy to be efficient. These devices can be divided into two sub-categories: Those that involve a feedback process in the flow, and those that generate longitudinal vorticity.

The former cathegory includes resonance based devices, such as cavities, ${ }^{3}$ impingement obstacles, ${ }^{4}$ and self-exciting wires. ${ }^{5}$ The cavity size, obstacles geometry and position, the wire diameter and tension are adjusted such that the associated frequency matches that of the main flow instability. Such devices are technologically simple and can achieve significant mixing enhancement.

The sub-category comprises devices which are used to interact with the longitudinal vortices developed in the incompressible mixing layer, ${ }^{1}$ thus destabilizing the KelvinHelmholtz vortices. Sandham and Reynolds ${ }^{6}$ proposed that such vortices also exist in supersonic mixing layers. However, experimental and computational studies have shown these Kelvin-Helmholtz cortices to have a three-dimensional character when the convective Mach number is high. Longitudinal vorticity generators can be in the form of small tabs or nozzle trailing edge geometry. The tabs are small obstacles placed inside the nozzle, very close to the trailing edge. They have been studied in subsonic and supersonic configurations by many authors. ${ }^{7-12}$ The action of tabs results in a strong distortion of the mixing layer and the generation of pairs of counter rotating longitudinal vortices, which induce in a strong mixing enhancement. Kim and 
Samimy ${ }^{13}$ proposed an alternative device, which is devoted to the supersonic configurations. The trailing edge is here shaped in order to generate longitudinal vorticity caused by a baroclinic torque in the case of under-expanded jets. The mixing layer thickness can be doubled in this way, without strong thrust loss.

Unfortunately, however, all of these passive devices are difficult to remove. Taking in account that in most cases the mixing enhancement is required only for specific flight phases (take-off or combat), active control systems are preferable.

Active devices are defined as devices that require energy to work efficiently. These devices can be classified depending on the actuator mechanism: Acoustic, mechanical, or pneumatic.

Acoustic devices comprise speakers located upstream of the jet flow or around the nozzle lip. This kind of actuator has been widely studied: Crow and Champagne, ${ }^{2}$ Raman and Rice, ${ }^{4,14}$ and Fiedler and Mensing, ${ }^{15}$ for instance. The speakers develop an excitation at a naturally unstable frequency of the flow. This forcing frequency effect on the flow is thought to be the opposite of the effects of longitudinal vorticity generators: Acoustic devices trigger the flow structures and enhance the development of naturally unstable modes. Zaman and Raman ${ }^{16}$ showed indeed that simultaneous use of tabs and speakers has relatively poor efficiency in terms of the resultant mixing enhancement. Finally, acoustic drivers are less efficient if the flow configuration is supersonic. Clemens and Mungal ${ }^{17,18}$ showed indeed that increasing the Mach number makes the mixing layer highly three-dimensional and poorly organized. Furthermore, acoustic wave propagation is inhibited by the supersonic mixing layer noise. Given these factors, forcing acoustically at a given frequency is not an effective mixing enhancement strategy for supersonic flow regimes.

The second active control system category concerns mechanical devices. Such devices comprise flapping actuators or piezoelectric elements. Wiltse and Glezer ${ }^{19}$ proved that a consistent mixing enhancement can be achieve using piezoelectric films located near a square subsonic jet exit. As piezoelectric elements work at a single given resonant frequency, the active control is realized via an amplitude modulation. Several azimuthal jet column instability modes can be driven using this system. Parekh et al. ${ }^{20}$ studied the effects of oscillating flaps located near subsonic or sonic rectangular jets. According to the frequency and amplitude excitation range, oscillating flaps generate a flapping, a vectoring or a bursting of the jet, except in the transonic regime, where only flapping occurs.

Finally, jet control can be performed using pneumatic devices. These systems are generally preferred to mechanical devices: On one hand they are easily adapted to real aircraft, and on the other hand they are more robust than mechanical devices regarding the temperature and vibration constraints in aircraft engines. Blowing axially or radially can achieve strong mixing enhancement. Papamoschou ${ }^{21}$ and Zaman and Papamoschou ${ }^{22}$ developed an axial blowing system. While a coaxial flow generally lowers the jet expansion by reducing the velocity shear, it is shown that under very specific con-

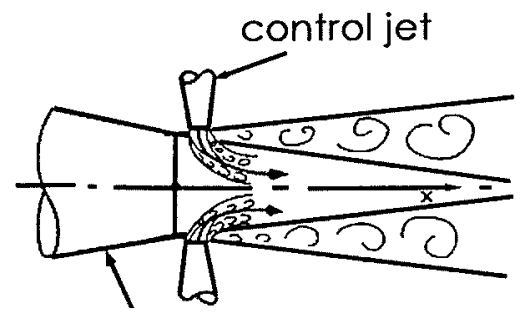

FIG. 1. Pneumatic jet mixing enhancement concept (Davis, 1982).

ditions, such a configuration can double the spreading rate. According to Papamoschou, ${ }^{21}$ the mixing enhancement process is linked to a shock instability in the imperfectly expanded coflow nozzle. Radially blowing systems were originally studied by Davis, ${ }^{23}$ in the supersonic regime. A schematic view of the concept is shown in Fig. 1. Regarding the mean velocity field, low flow-rate control jets (CJ) located just downstream of the main jet nozzle produce effects similar to those obtained with tabs. The mechanism responsible for mixing enhancement was initially thought to be based on longitudinal vorticity, generated by deflection of the $\mathrm{CJ}$ in the main flow direction. As demonstrated by the experiments of Parekh et al. ${ }^{20}$ and the direct numerical simulation of Freund and Moin, ${ }^{24}$ the CJ can be pulsed to optimize the actuator efficiency. In these studies, the main jet is transonic, and the CJ has a rectangular nozzle with a large aspect ratio. These pulsed control jets lead to a substantial mixing enhancement, strong jet asymmetry and large-scale vortices. Optimal mixing enhancement is achieved by forcing at a harmonic of the main jet column instability. Unfortunately, the $\mathrm{CJ}$ flow rate is very high in this configuration $(10 \%$ of the main jet flow rate).

Recently, Delville et al. ${ }^{25}$ furthered the pneumatic devices development. Direct numerical simulations, as well as experimental campaigns in subsonic and supersonic configurations have proved the efficiency of such devices. In this work, the CJs are small compared to the main jet, which leads to a cumulative CJ flow rate of less than $1 \%$ of the main jet flow rate. Examples of the velocity flow field and the longitudinal evolution of the average mixing layer thickness are presented in Fig. 2. Delville et $_{\text {al. }}{ }^{25}$ also showed that whether the configuration be subsonic or supersonic, the benefit of pulsed CJs, compared to steady ones at the same average flow rate is consistent, but not outstanding, even when the CJs are pulsed at a harmonic frequency of the jet column instability. For example, it was found that for $X / D=1.5$, the average mixing layer thickness with pulsed CJs is $16 \%$ greater than in the steady case. The difference between the pulsed and steady CJ is less important in the supersonic configuration. One hypothesis that may explain this behavior derives from the study of Zaman and Raman, ${ }^{16}$ where the simultaneous use of tabs and speakers was found to reduce the mixing enhancement. Indeed, if a CJ inputs longitudinal vorticity, it should completely change the manipulated flow organization. The manipulated flow would then completely loose its natural, well-identified unstable modes. The following question thus arises: by what kind of mixing enhancement mechanism do the CJs enhance mixing? Since Delville 


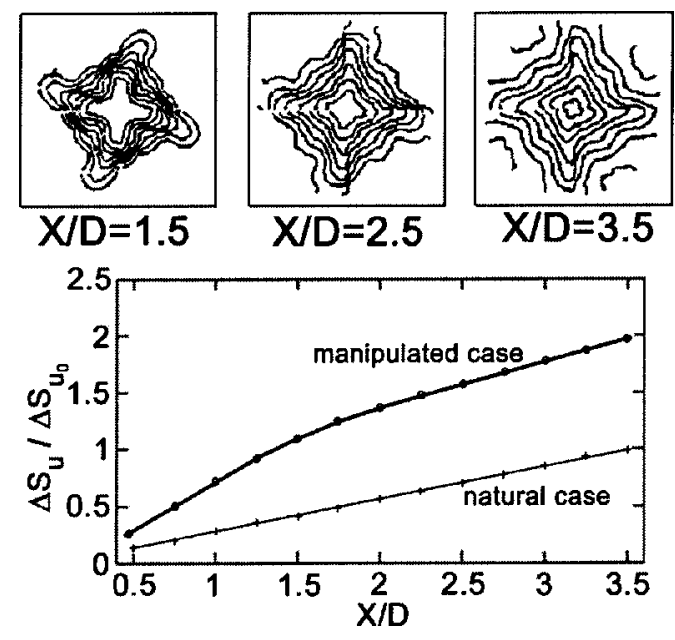

FIG. 2. Pneumatic jets mixing enhancement efficiency (Delville et al., 2000).

et $a l^{25}$ detected that mixing enhancement mainly occurs very near the main jet exit nozzle, it is obvious that further experiments are necessary in this region of the flow, which corresponds to the maximum of receptivity of the mixing layers.

The main objective of the present work is to experimentally investigate and then thus explain the interaction mechanism between one CJ and a supersonic mixing layer. This study constitutes a description and interpretation of the interaction mechanism, rather than a quantitative measurement of the mixing effect of this device.

We will focus, therefore, on the very near field interaction region (close the $\mathrm{CJ}$ ) where most of the process takes place.

Section II presents the experimental setup. In Sec. III, we put forward that the interaction between the CJ and the mixing layer produces a strong CJ penetration intermittency. This behavior involves dramatic changes of the manipulated mixing layer organization. Section III concerns laser Doppler velocimetry (LDV) measurements in the CJ-mixing layer interaction region. Finally, in Sec. IV we present some hypotheses to explain the observed behavior.

\section{EXPERIMENTAL SETUP}

\section{A. Wind tunnel and control jet}

Experiments were performed in the S150 supersonic wind tunnel located at the CEAT of Poitiers, France. This set-up consists of a $M=1.37$ supersonic jet surrounded by a subsonic entrained co-flow. Stagnation pressure and temperature for the main jet are respectively $3 \times 10^{5} \mathrm{~Pa}$ and $260 \mathrm{~K}$. The coflow velocity and stagnation temperature are $47 \mathrm{~m} / \mathrm{s}$ and $290 \mathrm{~K}$. The convective Mach number is $M_{C}=0.55$, and the convective velocity $U_{C}=230 \mathrm{~m} / \mathrm{s}$. The supersonic jet has the same static pressure as the subsonic co flow. The nozzle diameter is $D=50 \mathrm{~mm}$. The test section dimensions are 500 $\times 500 \mathrm{~mm}^{2}$. Lateral test section walls comprise large windows such that visualizations or laser Doppler measurements can be easily performed. A $200 \times 10^{5}$ Pa dry air tank supplies the main jet.

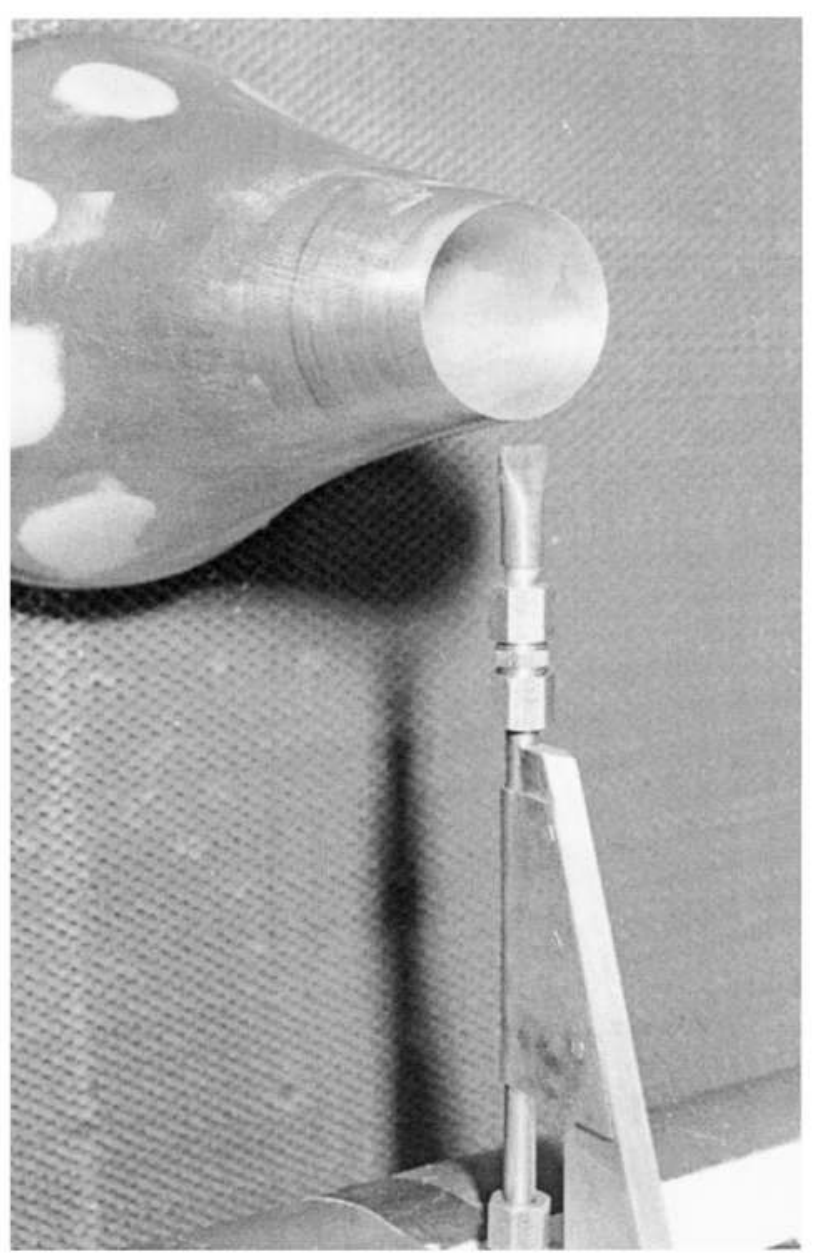

FIG. 3. Photography of the test section facility.

The CJ is supplied by the same dry air tank as the main jet and is placed at $X / D=0.1$ and $X / D=0.1$, as shown in Figs. 3 and 4. The CJ stagnation pressure is kept constant at $2.5 \times 10^{5} \mathrm{~Pa}$, which results in a velocity ratio $U_{\mathrm{CJ}} / U_{1}$ $\approx 0.89$. The CJ nozzle is convergent, and its exit is sonic. The geometry of the CJ used here corresponds to the more efficient among the geometries tested by Delville et al. $^{25}$ in supersonic conditions. It consists of a $11 \times 1 \mathrm{~mm}^{2}$ rectangular CJ. Fot our tests conditions, the mass flow-rate ratio between the $\mathrm{CJ}$ and the main supersonic jet is approximately $0.47 \%$.

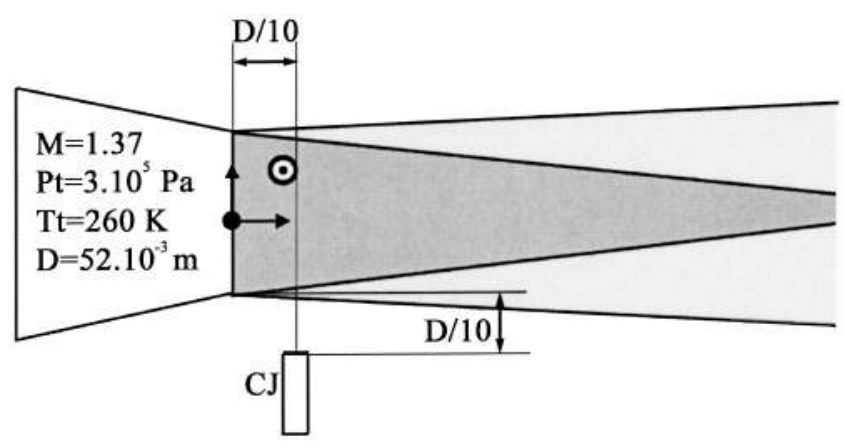

FIG. 4. Flow characteristics. 


\section{B. Flow visualizations and measurements techniques}

\section{Seeding techniques}

Flow visualizations and LDV measurements were effected using several seeding methods. For seeded CJ visualizations, we use $\mathrm{SiO}_{2}$ particles and a bypass system. According to the manufacturer data sheet and the results of Lammari, ${ }^{26}$ the average diameter of these particles is of the order of $4 \times 10^{-8} \mathrm{~m}$, but the seeding tank can contain agglomerates of the order of $4 \times 10^{-7} \mathrm{~m}$. Seeding is realized far upstream the CJ exit in order to obtain a homogeneous distribution. The bypass system ensures a balanced seeding. As the $\mathrm{SiO}_{2}$ particles did not bring high quality mixing layer visualizations, seeding of the mixing layer was performed using ethanol droplets injected in the upper and lower regions of the main settling chamber. The droplets size is strongly inhomogeneous and this seeding is certainly perfectible for further studies. Nevertheless, ethanol droplets do produce visualizations sufficient for qualitative observations.

Laser Doppler measurements were performed by seeding with $\mathrm{SiO}_{2}$ particles where each of the three flows (the $\mathrm{CJ}$, the main jet, and the coflow) were seeded separately. The CJ is seeded by the same method as for visualizations. The particle concentration is, however, lower for LDV measurements. The main jet is seeded by injection in the settling chamber and the surrounding coflow is seeded by injection through an annular injector. A multivalve system allows the seeding to be balanced.

\section{Visualizations apparatus}

Visualizations of the flow are obtained with an optical system of a Dantec PIV system. A laser sheet is generated from a $200 \mathrm{~mJ}$ Nd YAG pulsed laser beam. Using a set of mirrors and a cylindrical lens, the laser sheet can be positioned either in the symmetry plane of the main jet or in a cross section. The time of exposure is about $8 \mathrm{~ns}$, which is short enough to "freeze" the flow. Pictures are acquired on computer by use of a CCD camera. For each run we acquire 200 pairs of instantaneous side-view pictures. Each picture has $768 \times 484$ pixels, coded on 256 grayscale levels. Simple algorithms give well-converged average and rms pictures for each run. Moreover, as instantaneous pictures of a given pair are separated by a small time step, we can perform advanced picture analyses.

High-speed camera visualizations are also presented, these issuing from collaborative work with Spectra-Physics and LaVision experts. We used a UltraSpeedStar camera system from LaVision with 16 frames at a maximum frame rate of $1 \mathrm{MHz}$. The system integrates a pulsed DPSS laser from Spectra-Physics running up to $100 \mathrm{kHz}$. Sequences of 16 consecutive instantaneous pictures are acquired with a sampling frequency equal to $100 \mathrm{kHz}$. The size of these pictures is $640 \times 511$ pixels, with a 16 bit grayscale level. Due to experimental constraints, high-speed camera visualizations are performed in a free supersonic jet configuration, rather than a configuration comprising a coflow.

Figure 5 illustrates the three optical configurations used. Most of the snapshots presented in this paper are obtained in

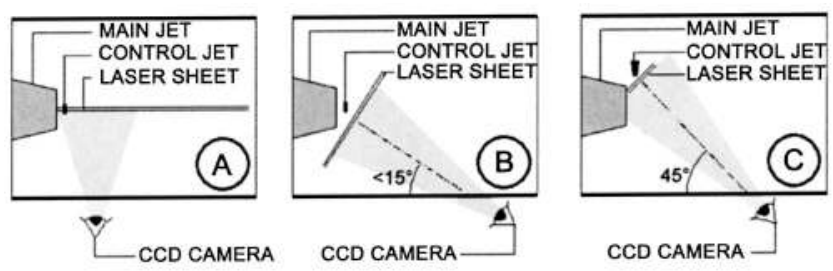

FIG. 5. Optical setups for flow visualizations.

configuration A. Configurations $\mathrm{B}$ and $\mathrm{C}$ are used for quasicross-sectional instantaneous views of the seeded CJ.

\section{Laser Doppler measurements}

Laser Doppler measurements are obtained using a fringe mode two-components Aerometrics system. The optical system comprises a $10 \mathrm{~W}$ nominal power laser source, a beam separator, a Bragg cell, emission and reception modules, filters and photo detectors. The optical arrangement is based on forward scatter principle. An Aerometrics DSA module analyzes the signal and stores the data to a computer. More than 1500 samples are acquired for turbulent stress measurements, and more than 50000 samples are used for spectral processing. LDV measurements are made with three seeding sources (main jet, coflow, and CJ). We try, in the present work, to lower as much as possible the seeding bias which may occur in supersonic free shear flow. The seeding method was derived from the work of Lammari ${ }^{26}$ and Lammari et al. ${ }^{27}$ (1996) who investigate the best seeding strategy in this kind of supersonic free shear flow. This method leads to acceptable seeding bias when used for the determination of Reynolds stresses. Concerning the effect of shock wave-seeding particle interaction it has been shown by Alem ${ }^{28}$ and Barre et al. ${ }^{29}$ that for the present range of Mach number ( $M$ $\approx 1.4$ ) the particle inertia effect when crossing a shock wave may be neglected in the context of the objectives of the present study, which are rather to describe the large scale structure organization in the interaction than to accurately measure the Reynolds stress tensor.

\section{FLOW VISUALIZATIONS}

\section{A. Snapshots and high-speed camera series}

Figure 6 gives an example of the CJ effect on the organization of the mixing layer structures. From Figs. 6(a) and 6(b), it can be observed when comparing the upper and lower (close to the CJ device) part of the mixing layer that when inactive, the CJ nozzle does not affect the main flow. The blowing $\mathrm{CJ}$ on the other hand completely changes the flow organization. Very large-scale structures appear downstream of the CJ impact. These structures seem to be mainly spanwise oriented, and look like Kelvin-Helmholtz vortices. The most striking result is the degree of organization of the manipulated mixing layer [see the lower part of Fig. 6(b)], in comparison with the nonmanipulated case. Visualizations of Fig. 6 show that the mixing enhancement process occurs mainly upstream of $X / D=1$, and seems to comprise spanwise vortex generation rather than streamwise vorticity. Indeed, longitudinal vorticity generation would make the mix- 


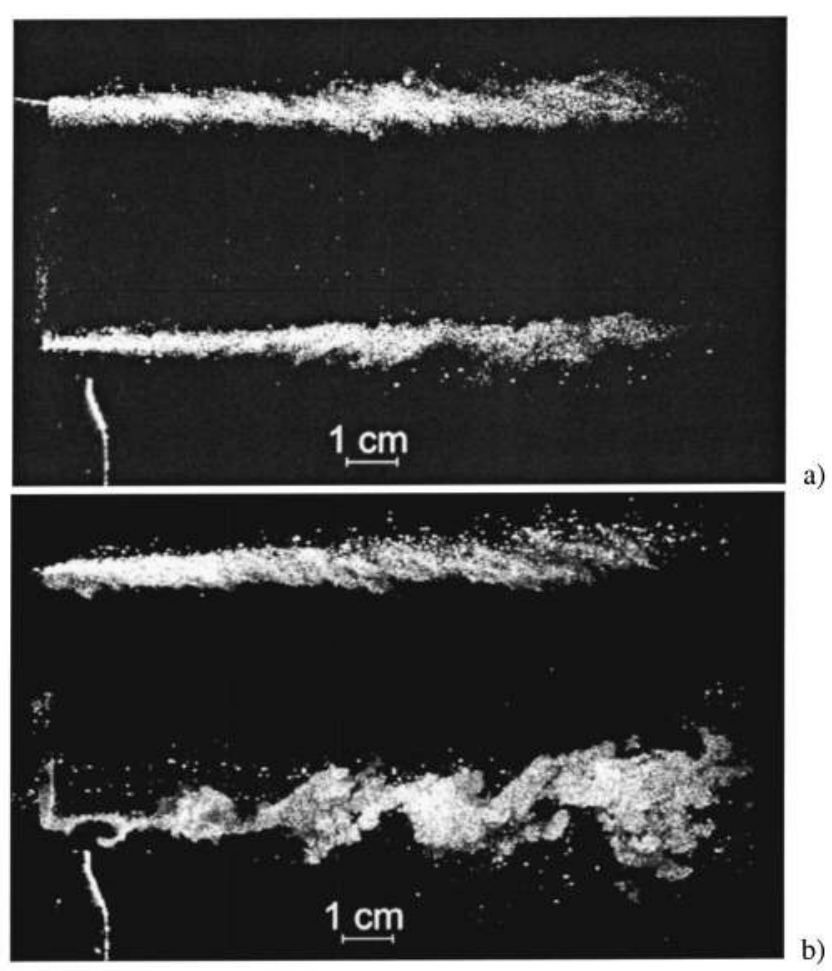

FIG. 6. Seeded mixing layer instantaneous views; (a): inactive CJ; (b): active CJ.

ing layer considerably three-dimensional, which is not consistent with the visualizations shown here.

As a first step in understanding of the origins of the manipulated mixing layer large scale structures, we depict on Fig. 7 two representative close-view snapshot examples of the seeded CJ. Large-scale spanwise vortices can also be seen on these visualizations. These structures seem to be caused by a very intermittent behavior in the near field of interaction between the $\mathrm{CJ}$ and the mixing layer. The CJ oscillates between deep penetration and completely blockedout stages.

Using a Lavision (C) high-speed camera system coupled with a Spectra Physic (C) laser source flashing continuously at a frequency of $100 \mathrm{kHz}$, we acquired several temporal visu-

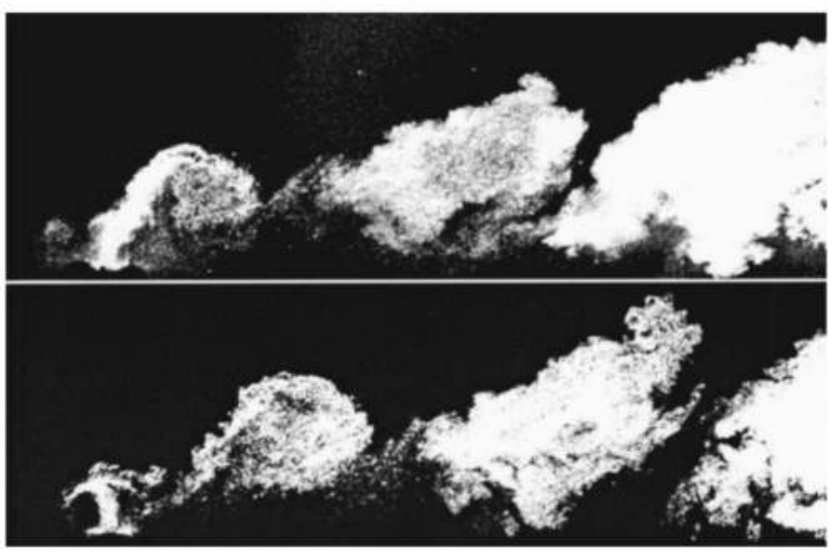

FIG. 7. Examples of seeded CJ instantaneous side views.

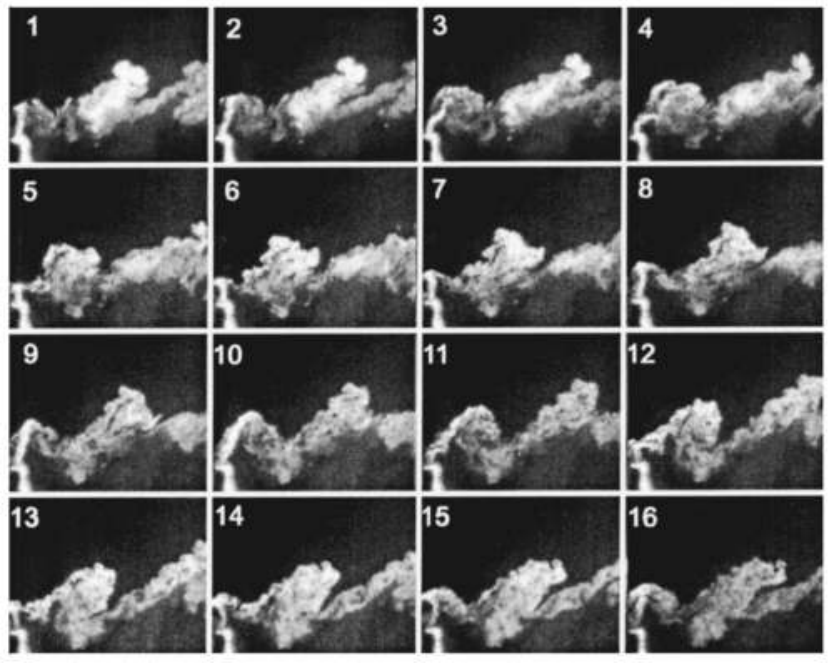

FIG. 8. High-speed camera side view sequence of the seeded CJ.

alization sequences. An example is shown in Fig. 8. Because of the very specific optical arrangement, for this part of the study the flow conditions comprise a main free jet manipulated by the same CJ as before.

The 16 snapshots are acquired at a $100 \mathrm{kHz}$. First, these visualization sequences illustrate that the CJ oscillations are periodic. Second, during this 16 snapshot sequence, roughly two CJ oscillation periods can be observed. This implies that the CJ flapping frequency is close to $13 \mathrm{kHz}$. The evaluation of the flapping frequency is analyzed in more detail in Secs. III $B$ and IV B.

Going back to the original visualization technique, we can also discuss the snapshots performed in a cross section of the seeded CJ. Instantaneous snapshots examples are given in Fig. 9 for cross sections $X / D=0.15$ and 0.3 . These cross sections are obtained using optical configurations $\mathrm{B}$ and $\mathrm{C}$, respectively, as described in Sec. II B and Fig. 5. However, these examples are not very representative, since the CJ flapping has a strong influence on the position of the CJ plume. Nevertheless, pictures in Fig. 9 reveal the generation of longitudinal vortices. Despite the very intermittent behavior of the CJ-mixing layer interaction, several cross-sectional snapshots reveal structures that are very similar to those generally found for the conventional configuration of a jet in a cross flow. The picture taken at $X / D=0.15$ shows several longitudinal vortices whose size is typically of the order of the CJ thickness. These structures look like those obtained
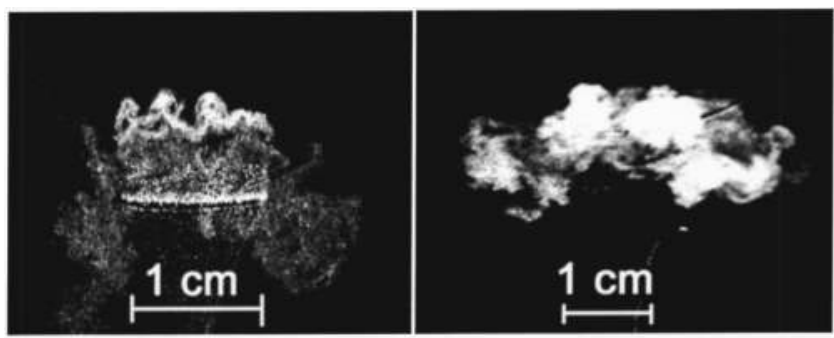

FIG. 9. Examples of the seeded CJ end views (left: $X / D=0.15$; right: $X / D=0.3)$ 


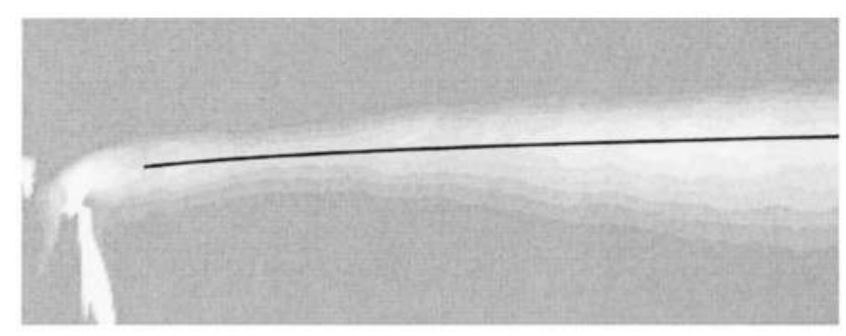

FIG. 10. CJ average trajectory illustration.

when a Dean instability occurs. Farther downstream, the visualizations reveal that the streamwise vortices are rearranged in a pair of counter-rotating structures, with a scale that matches the CJ span. Longitudinal vortices may be caused by the curvature of the CJ streamlines corresponding to the deep penetration stage.

As an intermediate conclusion, it is shown that the main feature of the CJ-mixing layer interaction is a periodic penetration process. This behavior has a strong influence on the manipulated mixing-layer structures. Nevertheless, streamwise vortices are still developed downstream of the CJ. The mixing enhancement seems to be linked to the penetration instability, rather than with the longitudinal vorticity as originally thought. The next paragraph focuses on the evaluation of the manipulated mixing layer structure-spacing and the $\mathrm{CJ}$ flapping frequency.

\section{B. Two point correlations}

In order to determine the manipulated mixing layer structure-spacing, we extract two point correlations using the snapshots database. Supposing that this spacing is equal to the inter-structure spacing in the CJ plume, the computations are performed on the seeded CJ side views.

The first step involves extraction of the grayscale levels from the CJ average trajectory on the images. This trajectory is defined as the curve of maximum grayscale in the average CJ side-view, and is illustrated in Fig. 10. Row-column coordinates of pixels on the average $\mathrm{CJ}$ trajectory are written $\left[i_{0}(x) ; j_{0}(x)\right]$, where $x$ denotes the curvilinear coordinate along the curve. Let $G^{\prime}(x, m)$ be the fluctuations of the grayscale level for the pixel $\left[i_{0}(x) ; j_{0}(x)\right]$ on a snapshot number $m$, the correlation function for image number $m$ is thus

$$
r_{G G}(\Delta x, m)=\frac{\left[G^{\prime}(x, m) \cdot G^{\prime}(x+\Delta x, m)\right]_{X}}{\left[G^{\prime}(x, m)^{2}\right]_{X}},
$$

where $[\cdot]_{X}$ denotes an average over $x$. Since we have several sets of 200 snapshot pairs, a time-averaged correlation function is obtained:

$$
R_{G G}(\Delta x)=\left[r_{G G}(\Delta x, m)\right]_{M},
$$

where $[\cdot]_{M}$ denotes an average over the $M$ snapshots.

The $R_{G G}$ correlation function plotted in Fig. 11 reaches a local maximum for $\Delta x=\lambda_{\mathrm{CJ}} \approx 20 \mathrm{~mm}$. This value corresponds to the average longitudinal wavelength of the structures in the CJ plume. This wavelength can be compared with $\lambda_{\mathrm{KH}}$, the Kelvin-Helmholtz inter-structure spacing in the corresponding nonmanipulated mixing layer. According

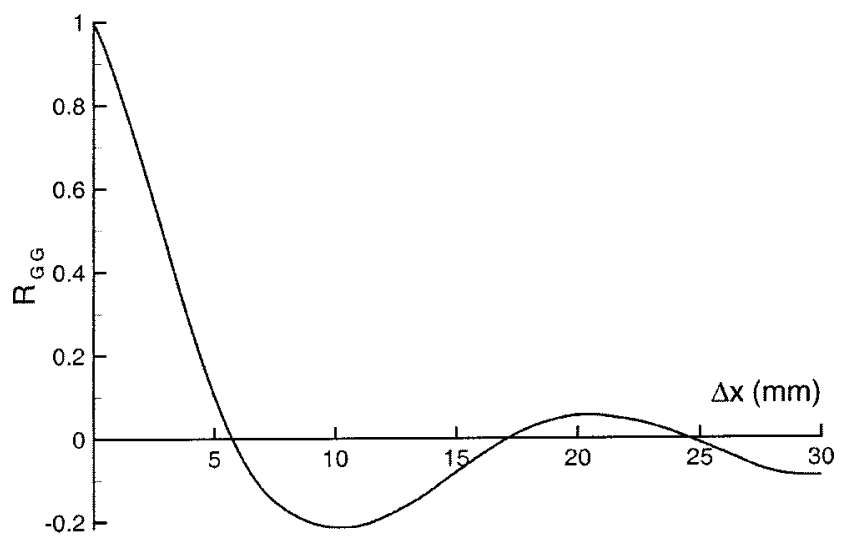

FIG. 11. Auto-correlation function in the CJ average trajectory.

to Bellaud et al. ${ }^{30}$ the local Strouhal number $S t_{M L}$ is about 0.2 in the nonmanipulated mixing layer, and the vorticity thickness $\delta_{\omega}$ is $3 \mathrm{~mm}$ at the CJ impact location. Then, the Kelvin-Helmholtz theoretical wavelength of the mixing layer at the CJ location is about

$$
\lambda_{\mathrm{KH}} \approx \delta_{\omega} / S t_{M L} \approx 15 \mathrm{~mm} \text {. }
$$

This, combined with a theoretical $\mathrm{KH}$ structure convection velocity:

$$
U c_{t h}=\frac{a_{2} U_{1}+a_{1} U_{2}}{a_{1}+a_{2}} \approx 230 \mathrm{~m} / \mathrm{s},
$$

gives a frequency

$$
f_{\mathrm{KH}}=U_{c t h} / \lambda_{\mathrm{KH}} \approx 15.3 \mathrm{kHz} \text {. }
$$

It is clear that the detected wavelength $\lambda_{C J}$ in the CJmixing layer interaction is of the order of magnitude of $\lambda_{\mathrm{KH}}$. Then, at this stage of the study, a link between the CJ flapping and the Kelvin-Helmholtz type structures in the initial mixing layer may be supposed, if not proven. To investigate this possibility we will try to obtain from the two-point correlation, a convective velocity for the structures downstream of the CJ. From this convective velocity we will be able to compute a flapping frequency for the $\mathrm{CJ}$ and then compare it to $f_{M L}$.

The following method is used to estimate the convective velocity of the CJ structures. Since we have 200 pairs of instantaneous pictures, we can cross correlate between the two images of a given pair. The PIV system gives the time difference between the two images: $\Delta t=20 \cdot 10^{-6} \mathrm{~s}$.

Let $G_{A}^{\prime}(x, m)$ and $G_{B}^{\prime}(x, m)$ be the fluctuations of the grayscale level of a pixel on the CJ average trajectory for images $A$ and $B$ from the pair of instantaneous views number $m$. The cross correlation function is then

$$
r_{A B}(\Delta x, m)=\frac{\left[G_{A}^{\prime}(x, m) \cdot G_{B}^{\prime}(x+\Delta x, m)\right]_{X}}{\sqrt{\left[G_{A}^{\prime}(x, m)^{2}\right]_{X}\left[G_{B}^{\prime}(x, m)^{2}\right]_{X}}} .
$$

This variable can be time averaged

$$
R_{A B}(\Delta x)=\left[r_{A B}(\Delta x, m)\right]_{M} .
$$

In order to read directly a convective velocity, the crosscorrelation function is plotted as a function of $\Delta x / \Delta t$ in Fig. 


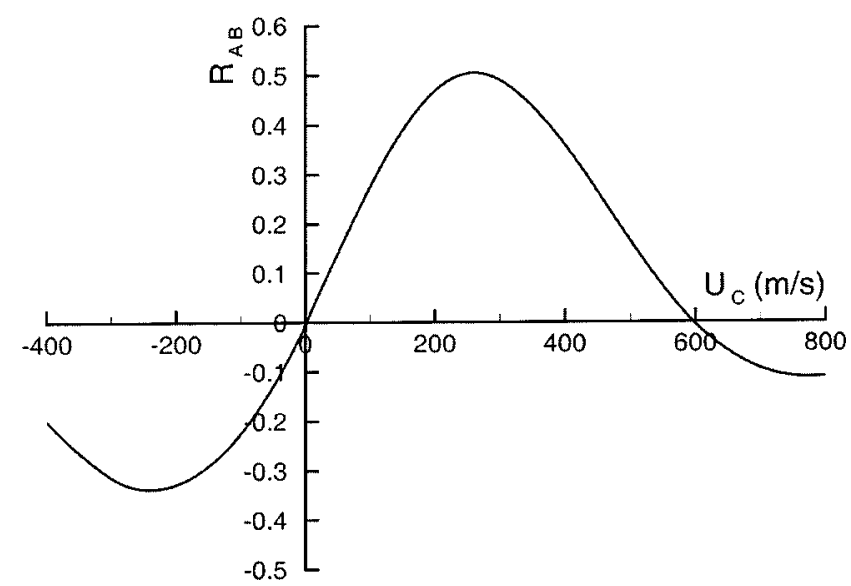

FIG. 12. Cross correlation function in the CJ plume.

12. This diagram leads to a convective velocity of $260 \mathrm{~m} / \mathrm{s}$, which is just a little bit higher $(\approx 13 \%)$ than the theoretical convective velocity in the nonmanipulated mixing layer

$$
U c_{\mathrm{th}}=\frac{a_{2} U_{1}+a_{1} U_{2}}{a_{1}+a_{2}} \approx 230 \mathrm{~m} / \mathrm{s} .
$$

The CJ flapping frequency can now be estimated

$$
f_{\mathrm{CJ}}=U_{c} / \lambda_{\mathrm{CJ}} \approx 13 \mathrm{kHz} \text {. }
$$

This flapping frequency determined from the visualization analysis seems quite close $(\approx 15 \%)$ to the theoretical Kelvin-Helmholtz frequency of the initial mixing layer at the CJ location.

Since the mechanism responsible of the CJ intermittent penetration is not well understood, we can propose two hypotheses.

First, as the CJ size is quite small, it is possible that the penetration depends on the presence of a Kelvin-Helmholtz structure in front of the CJ exit. Indeed, if the adverse pressure gradient were sufficiently strong just upstream of a Kelvin-Helmholtz rollup, the penetration of the CJ would be reduced. On the other hand, when the CJ nozzle is upstream or downstream of a Kelvin-Helmholtz roll up, the penetration is enhanced. A schematic description of this hypothesis is given in Fig. 13(a). This mechanism implies that the Kelvin-Helmholtz rollups generate very strong pressure fluctuations. Such behavior has been evidenced by the DNS results of Lardeau et al., ${ }^{31}$ in a low Reynolds subsonic flow, where Kelvin-Helmholtz rollups are very well organized spatially and temporally.

The other hypothesis is based on an intrinsic instability of the interaction between the $\mathrm{CJ}$ and the annular mixing layer. This instability may be due to the generation of a highpressure region just upstream of the CJ, caused by a local downward deviation of the main jet. When the pressure upstream of the CJ is sufficiently strong (i.e., at least equal to the CJ stagnation pressure), the high-pressure zone is convected downstream, stopping the penetration of the CJ. Fig-

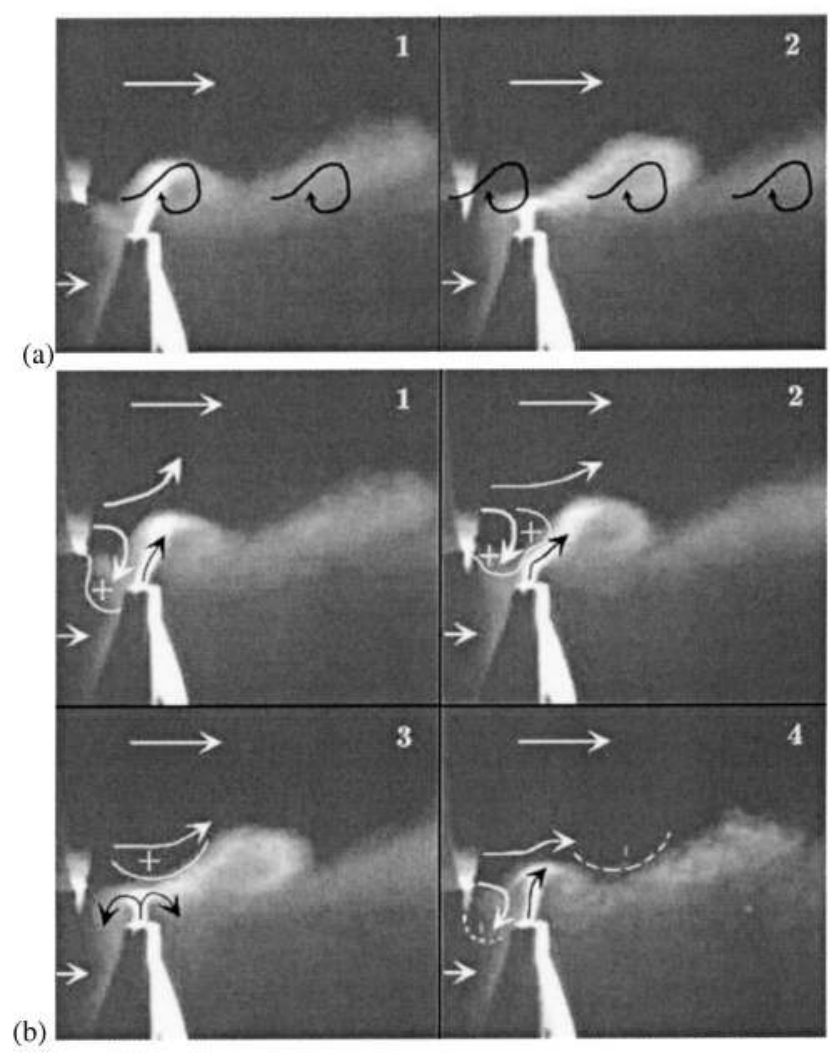

FIG. 13. Schematic description of the two mechanisms responsible for the CJ penetration intermittency.

ure 13(b) illustrates this hypothesis. In this scenario, the typical frequency of the instability should adapt itself to the local mixing layer frequency $f_{M L}$.

A comment should be made concerning the second hypothesis: since a portion of high speed fluid seems to be deviated downward, just along the upward CJ stream, an absolute instability (AI) could play a significant role in the CJ destabilization process. Nonintrusive velocity measurements detailed in Sec. IV help to settle on the AI hypothesis.

Additional experiments were performed to test these hypotheses. Instantaneous visualizations were performed, increasing the stagnation pressure of the $\mathrm{CJ}$ up to 10 $\times 10^{5} \mathrm{~Pa}$. Two representative instantaneous images for a CJ stagnation pressure of $10 \times 10^{5} \mathrm{~Pa}$ are shown in Fig. 14 . Since the CJ nozzle is convergent, it is strongly underexpanded for this regime. A normal shock wave occurs near the main mixing layer. Downstream the recompression shock wave, the CJ is flapping. Except the fact that the CJ structures are less organized than with a lower CJ stagnation pressure, no difference in the nature of the interaction appears. Taking into account that the stagnation pressure of the main jet is $3 \times 10^{5} \mathrm{~Pa}$, this result tends to disqualify the first hypothesis. This does not however prove that the second hypothesis is correct but just that it fits the present experimental results.

Finally, it can be noticed that the CJ flapping behavior should not be induced by an organ-pipe resonance in the CJ nozzle: The CJ flapping has indeed been observed with vari ous CJ nozzle geometries, main jet Mach number and CJ 


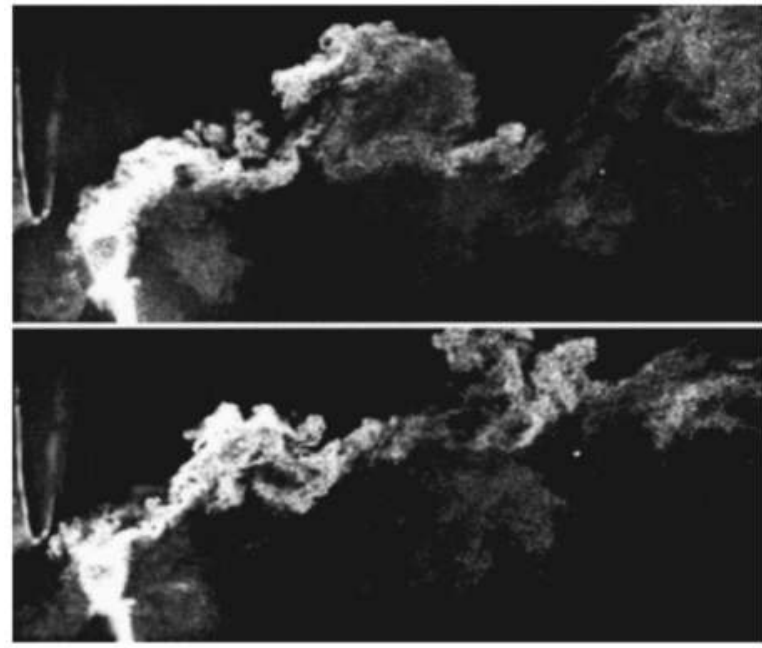

FIG. 14. Examples of seeded CJ instantaneous side views for a CJ stagnation pressure of $10 \times 10^{5} \mathrm{~Pa}$.

pneumatic setup. $^{32}$

In order to increase our knowledge of this interaction LDV measurements of turbulent stresses and CJ flapping frequency will be presented in the next section.

\section{TURBULENT FIELD AND CJ FLAPPING LDV STUDY}

In this section we discuss the LDV measurements made for 106 positions in the $(x, y)$ plane. These points are not regularly distributed: the mesh is finer near the impact region than further downstream. The measurements are made for the $(U, V)$ components.

\section{A. Average and turbulent flow fields}

Figure 15(a) presents the mean velocity field in the plane where the nondimensional velocity $\left(U-U_{2}\right) / \Delta U$ is plotted. This diagram demonstrates on one hand a strong main jet mixing layer distortion near the $\mathrm{CJ}$, and on the other hand a significant mixing enhancement achieved at $X / D>0.3$. We notice also that the $\mathrm{CJ}$ induces a slightly lower speed region in the main supersonic jet. This may be evidence of a shock wave generated in the supersonic flow when the $\mathrm{CJ}$ is at the deep penetration stage. We were not able to identify such a shock presence.
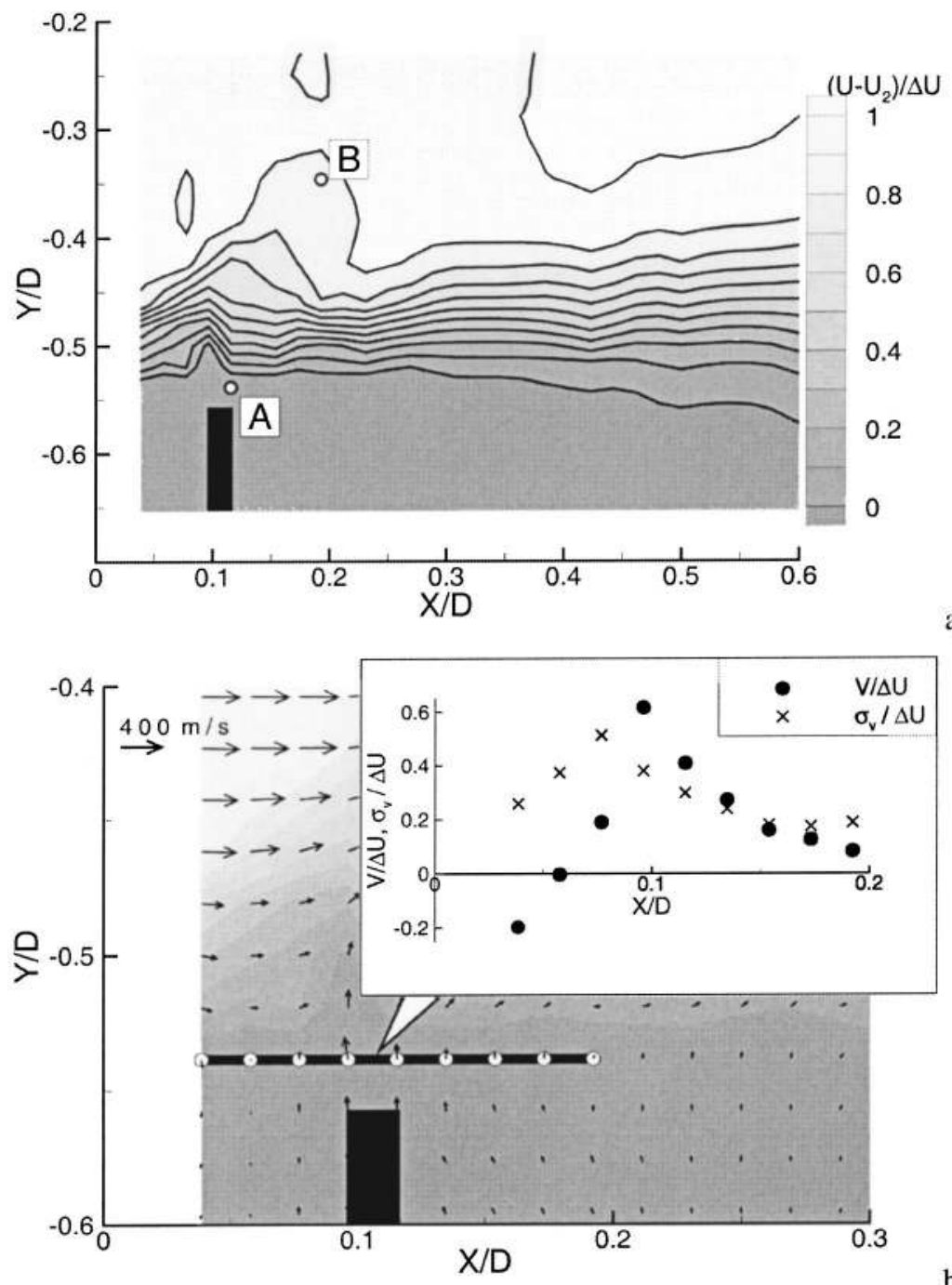

FIG. 15. (a) Mean velocity field in plane $(x, y)$; $\mathrm{CJ}$ is symbolized by a black rectangle; points A and B display locations where quadrant and spectral analyses were performed; (b) close-up view near the CJ exit. 

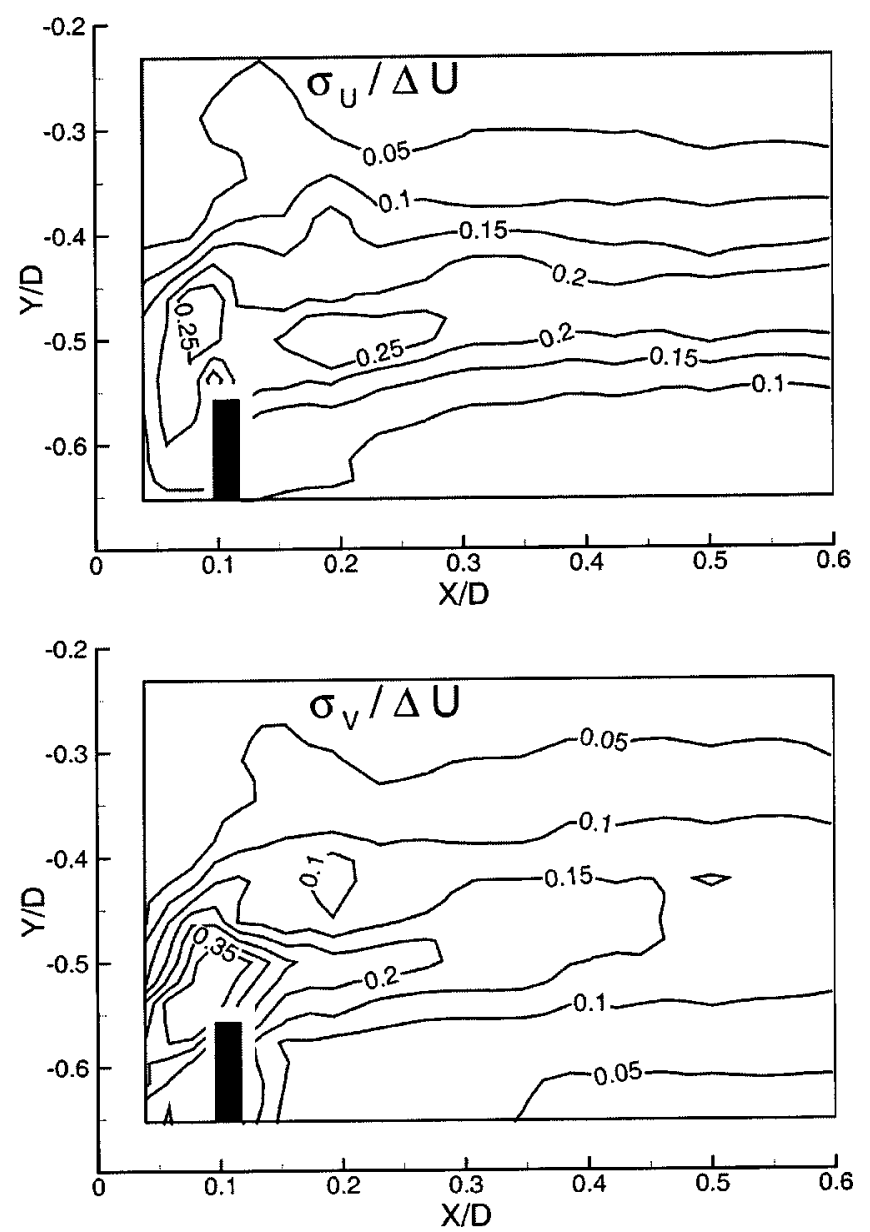

FIG. 16. $U$ and $V$ component fluctuations in the $(x, y)$ plane.

Otherwise, zooming in on the CJ zone, we detect strong $\mathrm{V}$ component variations, as shown in Fig. 15(b). Indeed, for $X / D=0.1$ and $Y / D=-0.54$, that is to say $2 \mathrm{~mm}$ above the CJ, we obtain $V / \Delta U=0.62$ and $\sigma_{V} / \Delta U=0.38$, whereas for $X / D=0.04$ and $Y / D=-0.54$, that is to say only $3 \mathrm{~mm}$ upstream, we have $V / \Delta U=-0.20$ and $\sigma_{V} / \Delta U=0.26$. Even if these are averaged values in a very unstable region of the flow, this denotes that a strong counter-flow shear layer exists between the $\mathrm{CJ}$ and a part of the main jet that is deviated downward just upstream of the CJ. The ratio between the velocity difference and the velocity sum between these two points is equal to 1.95. According to Huerre and Monkewitz ${ }^{33}$ the absolute instability threshold is crossed when the velocity ratio is higher than 1.315 . As suspected at the end of Sec. III, an absolute instability process may play a part in the CJ intermittency mechanism.

Root mean square fluctuations of the $u$ and $v$ velocity components are shown in Fig. 16. It can be noticed that both $\sigma_{U}$ and $\sigma_{V}$ are very strong near the CJ impact. In a nonmanipulated case, and with the same measurements setup as ours, Bellaud et al..$^{30}$ obtained maximum values of $\sigma_{U} / \Delta U$ and $\sigma_{V} / \Delta U$ close to 0.15 and 0.08 , respectively. In our case, the CJ produces values $\sigma_{U}$ and $\sigma_{V}$ two to four times greater than in the nonmanipulated case. This occurs mainly in the transverse direction $(y)$ where the flapping of the CJ leads to a huge level for transverse velocity fluctuations $\left(\sigma_{V}\right)$. Nev-

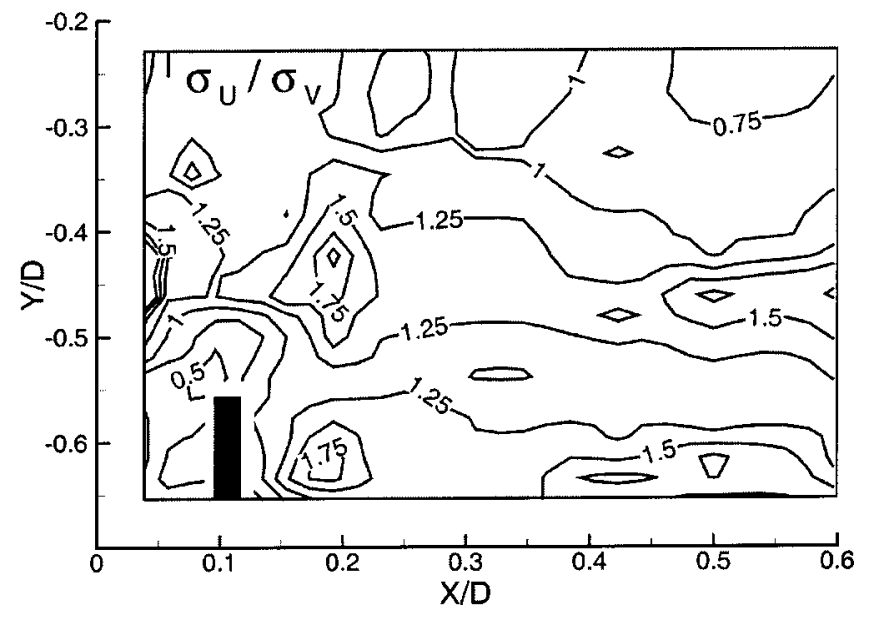

FIG. 17. Anisotropy parameter in the $(x, y)$ plane.

ertheless, for $X / D>0.5$, the effects of the CJ on the $\sigma_{U}$ and $\sigma_{V}$ fields seems to progressively vanish, revealing a return to the natural annular mixing layer equilibrium and the end of the mixing enhancement process.

As the CJ appears to severely change the mixing layer equilibrium, it is interesting to analyze the evolution of an anisotropy factor. The anisotropy factor, defined in the present study as the ratio $\sigma_{U} / \sigma_{V}$ is plotted in Fig. 17. Compared with the nonmanipulated case, for which $\sigma_{U} / \sigma_{V}$ follows a hat-shaped profile across the mixing layer with a maximum value close to 2 in the center of the mixing layer (Bellaud et al. ${ }^{30}$ ), results of Fig. 17 show that the anisotropy factor in the manipulated case presents a very singular shape. Indeed, the anisotropy factor does not exceed 1.5 for $X / D$ $<0.4$, except just upstream and downstream the CJ, where maximum values of $\sigma_{U} / \sigma_{V}$ locally exceed 2 . Moreover, for $X / D>0.25$, the anisotropy factor tends to increase again with $X$, tending to come back to values found in the nonmanipulated case. It is clear that the large values of $\sigma_{V}$ due to the intermittent behavior of the $\mathrm{CJ}$ have major influence on the anisotropy factor $\sigma_{U} / \sigma_{V}$.

The effects of the CJ on the turbulent shear stress field are also important, as shown in Fig. 18. Whereas Bellaud

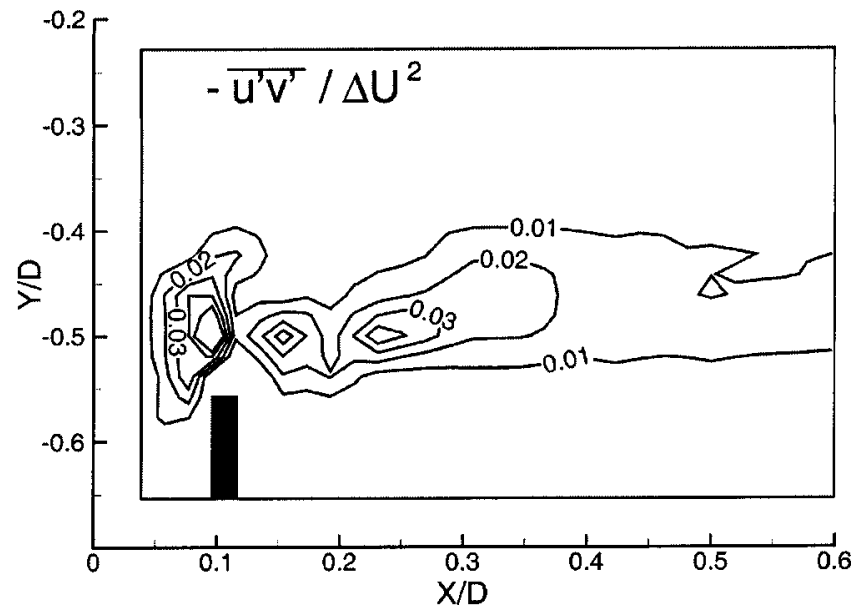

FIG. 18. Turbulent shear stress distribution in the $(x, y)$ plane. 


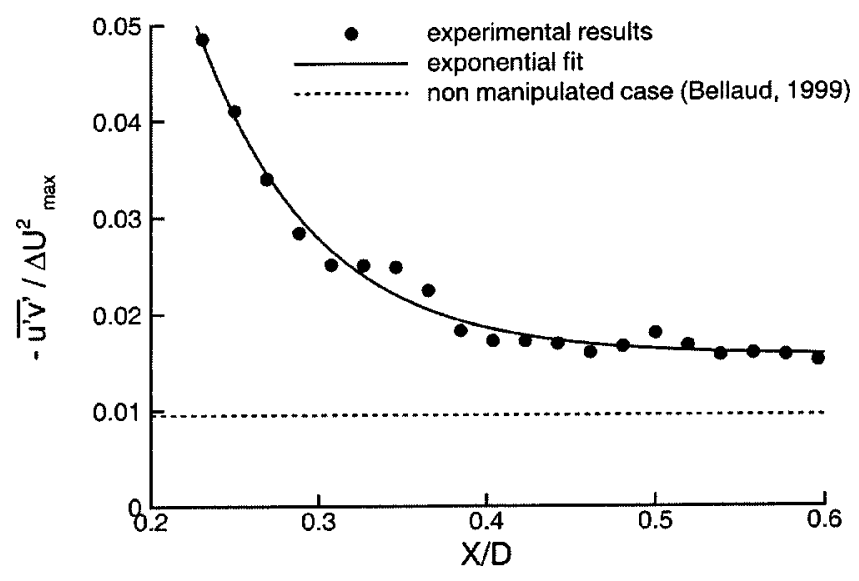

FIG. 19. Maximum shear stress longitudinal evolution.

et $a l{ }^{30}$ found the maximum value of $-\overline{u^{\prime} v^{\prime}} / \Delta U^{2}$ not to exceed 0.01 in the nonmanipulated case, we detect that the turbulent shear stress can reach values up to 0.05 near the impact region. These high values are consistent with the fact that the CJ is flapping in this region of the flow. Indeed, when in the penetration stage we expect $u^{\prime}<0$ and $v^{\prime}>0$, whereas when the $\mathrm{CJ}$ is stopped at the boundary of the main jet we should have $u^{\prime}>0$ and $v^{\prime}<0$. However, and like other variables analyzed just before, maximum values of $-\overline{u^{\prime} v^{\prime}} / \Delta U^{2}$ across the manipulated mixing layer tend to decrease downstream of the impact region. This behavior is demonstrated by Fig. 19, where it is obvious that the maximum turbulent shear stress decreases exponentially as $X / D$ increases. However, it should be observed that the rate of decrease is quite rapid. A quasi-asymptotic stage is reached by $X / D=0.5$.

According to the results shown above, the CJ produces important modifications on the mixing layer turbulence properties. The fluctuations of $u$ and $v$ velocity components are strongly increased, the anisotropy factor is reduced and the turbulent shear stress is boosted. However, the effects of the CJ remain local. For the most downstream locations where LDV measurements where performed, all the measured parameters return to values close to a nonmanipulated case, except that the mixing layer thickness is enhanced. This be-

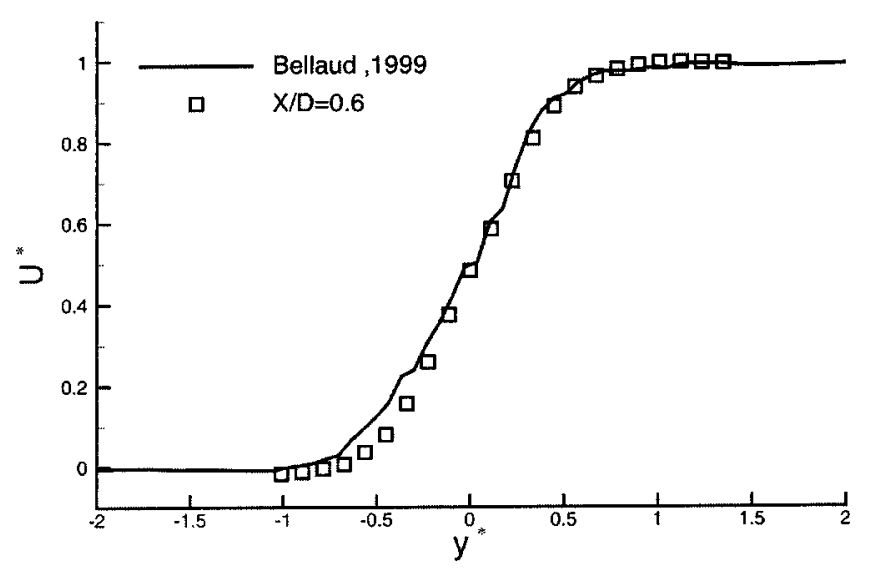

FIG. 20. Mean velocity profile comparison between manipulated and nonmanipulated mixing layer at $X / D=0.6$.

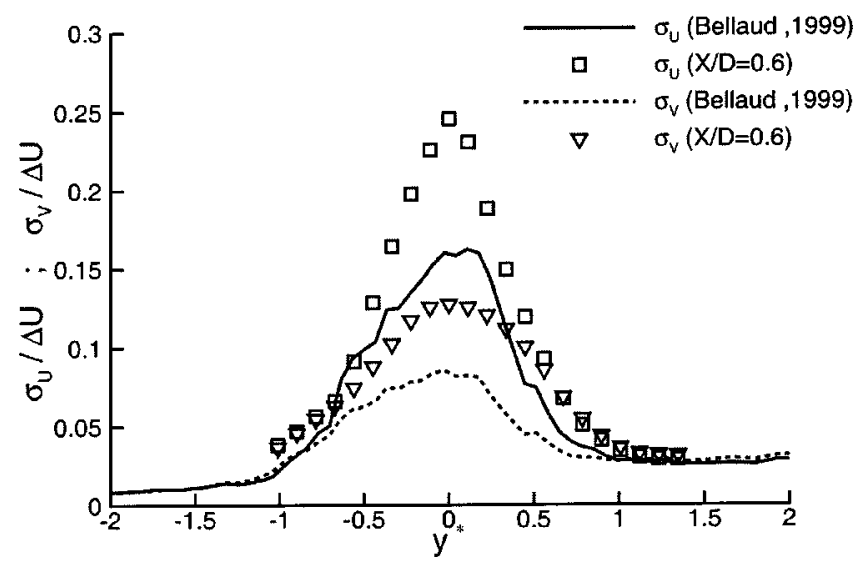

FIG. 21. $U$ and $V$ components velocity fluctuations profile for the manipulated and nonmanipulated mixing layer at $X / D=0.6$.

havior is consistent with the fact that mixing enhancement is localized very close to the $\mathrm{CJ}$ impact region, that is for $X / D<0.3$.

In order to further the discussion about the manipulated mixing layer relaxation downstream of the CJ, we compare results obtained in the present study for $X / D=0.6$ to those obtained by Bellaud et al. ${ }^{30}$ in the corresponding nonmanipulated case. Plots are drawn using the nondimensional variable $y^{*}$, defined as

$$
y^{*}=\frac{y-y_{\mathrm{ref}}}{\delta_{\omega}},
$$

where $U^{*}\left(y_{\text {ref }}\right)=0.5$ and $\delta_{\omega}$ is the vorticity thickness for each case. Mean velocity, velocity fluctuations, anisotropy factor and turbulent shear stress profiles are shown in Figs. 20-23.

Regarding the nondimensional mean velocity profiles, it appears that the differences between the nonmanipulated and the manipulated case are very small. From this point of view, the CJ effects have already completely disappeared for $X / D=0.6$. Concerning the anisotropy factor $\sigma_{U} / \sigma_{V}$ we notice that peak values are very close between the nonmanipulated and the manipulated case. On the contrary, there is a

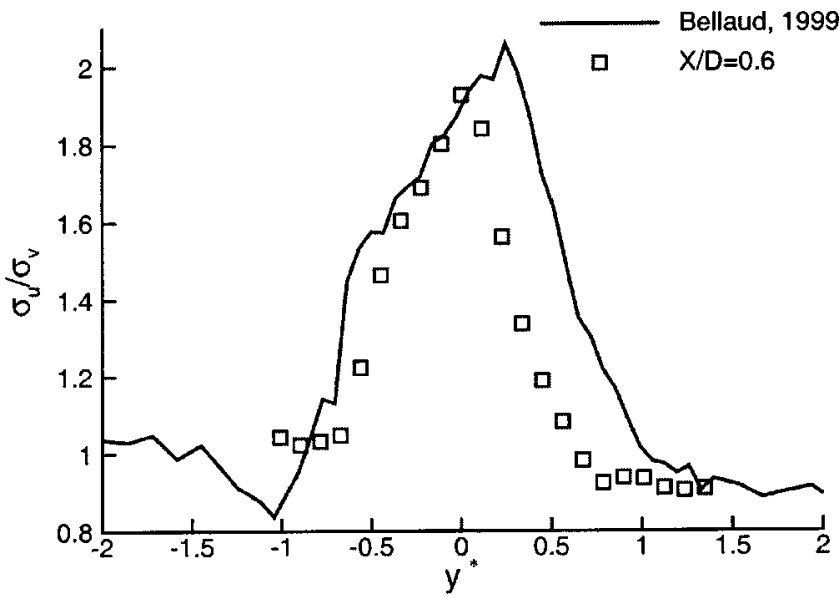

FIG. 22. Anisotropy factor profile comparison between manipulated and nonmanipulated mixing layer at $X / D=0.6$. 


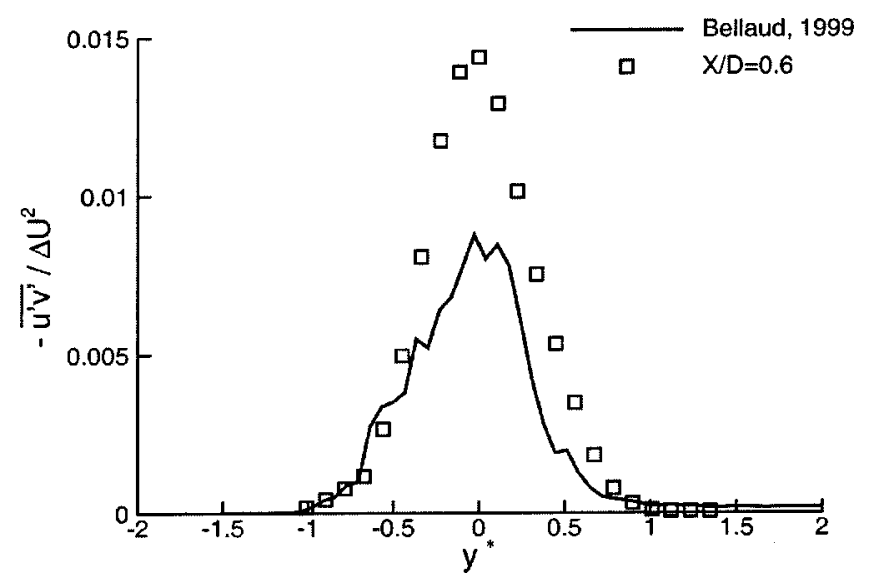

FIG. 23. Turbulent shear stress profile comparison between manipulated and nonmanipulated mixing layer at $X / D=0.6$.

difference in the shape of the $\sigma_{U} / \sigma_{V}$ evolution for $y^{*}>0$. In this high-speed region, the difference between the two cases may reach up to $50 \%$ in some places near $0.3<y^{*}<0.4$. It seems that in the high-speed part of the flow, return to nonmanipulated equilibrium is not completely achieved, perhaps due to the intermittency of the CJ, which may create a fluctuating shock wave on the high-speed side which would influence significantly the anisotropy factor. However, velocity fluctuations and turbulent shear stress profiles reveal that the CJ signature is still noticeable near $X / D=0.6$. Nevertheless, the shape of these profiles is not very different from the nonmanipulated case. The fundamental difference between the manipulated and the nonmanipulated cases is found in the amplitude.

As an intermediate conclusion we can argue that LDV measurements presented in this paragraph bring much infor- mation concerning the manipulated mixing layer status and the flow properties near the impact region.

\section{B. Spectral analysis}

The LDV results presented above bring a lot of information concerning the mean and turbulent manipulated flow field. In this section, we try to obtain some further knowledge of the flow organization.

First, when analyzing LDV data for several points of the measurement mesh, we noticed strange distributions of instantaneous bursts. In particular, very near the CJ impact on the main mixing layer, distribution of instantaneous velocity data reveal two or more distinct stages. One of the more outstanding examples is given in, for the point $A$, as defined in Fig. 15. This point is situated in the "wake region" of the $\mathrm{CJ}$, that is just downstream of the injection and on the lower speed side of the manipulated mixing layer. For this position, the turbulent shear stress satisfies $\overline{u^{\prime} v^{\prime}}>0$. Figure 24 shows a quadrant instantaneous representation of all the velocity events obtained in an LDV run at point A. The quadrant representation is accompanied by corresponding $U$ and $V$ velocity histograms (see Fig. 24). The fact that both $U$ and $V$ components vary more that $200 \mathrm{~m} / \mathrm{s}$ and that points in the quadrant representation are arranged in three groups reveals that the CJ intermittent penetration behavior (see Sec. III) is clearly detected by LDV measurements. From Fig. 24 it appears that most of the time the $\mathrm{CJ}$ is moving with a negative velocity $U$ and that when it penetrates the flow two kinds of event are observed. One with negative $U$ velocity and another one with positive $U$ velocity. This behavior seems consistent with flow visualizations presented earlier in this paper.
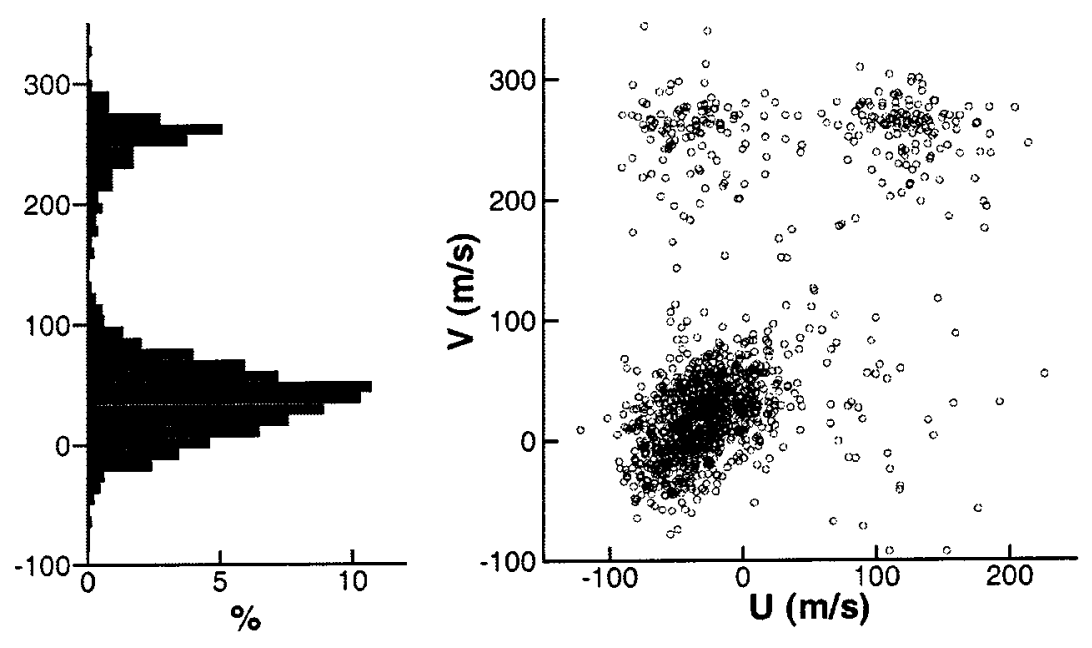

FIG. 24. Quadrant $(U, V)$ view for a run acquired at point $\mathrm{A}$ (cf. Fig. 15).

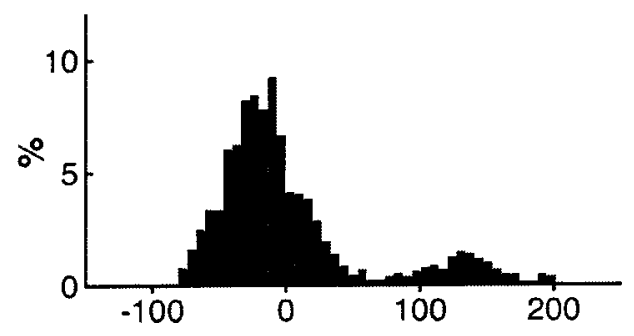




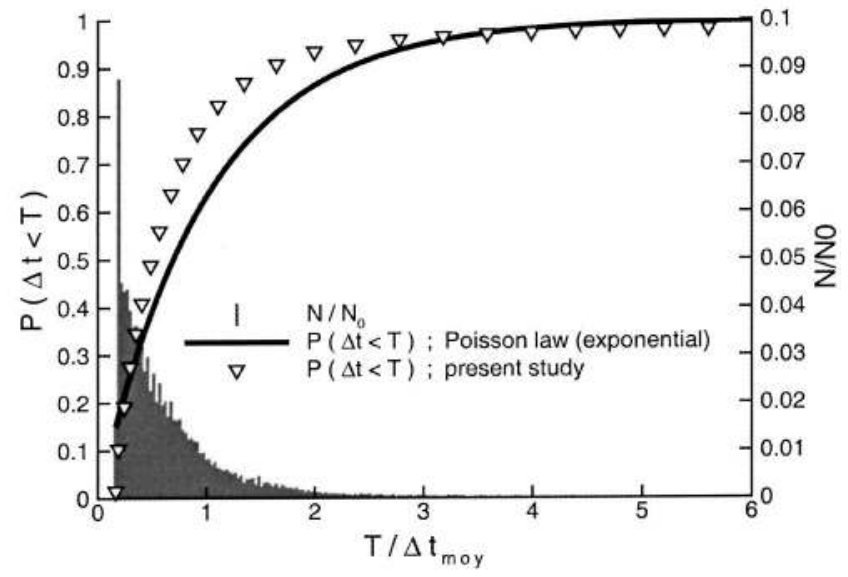

FIG. 25. Probability density function and histogram on time lag between two consecutive bursts.

Given the fact that the CJ intermittent penetration can also be observed with an LDV system, we planned to acquire longer runs in order to evaluate the CJ flapping frequency.

The first step is to choose a point in the $(x, y)$ plane where the average LDV sampling frequency is high and the fluctuations energetic enough. We choose to make the measurements at point B, which is represented in Fig. 15. This point is located in a high-speed region of the flow, which allows an average sampling rate up to $13 \mathrm{kHz}$, and the $U$ velocity fluctuations for this point are here of order $\sigma_{U} / \Delta U \approx 0.1$, which is sufficient to reveal information in the spectral domain.

The second step is to check that the sampling is made randomly and does not depend on flow events. The randomness of sampling is examined by analyzing the time intervals between two consecutive bursts. Let $T$ be an arbitrary time lag, and $P(\Delta t<T)$ the probability that the time lag $\Delta t$ is lower than $T$. According to Gaster and Roberts, ${ }^{34}$ the probability function $P(\Delta t<T)$ should follow a Poisson law. A comparison between the $P(\Delta t<T)$ function and the corresponding Poisson law is given in Fig. 25. The difference between our experimental results and the theoretical curve cannot be neglected. The following spectral analysis is then biased by the fact that our signal is not truly randomly sampled. Although the quality of the signal can be improved, the overall $P(\Delta t<T)$ shape seems to be close enough to a Poisson law to further the spectral analysis. In order to check that the sampling does not depend so much on flow events, we draw the quadrant distribution of burst in a velocity-time representation (see Fig. 26). No noticeable link between the sampling and the measurement can be made, which means that the burst time of arrival does not depend on the measured velocity.

To generate the LDV signal power spectra, we use the correlation method for which a detailed explanation can be found for instance in Bell. ${ }^{35}$ This method consists in building the auto-correlation function using discrete time intervals with a small allowance. The smaller the time interval size is, the smaller the number of events taken into account for each value of the correlation. In our case, we choose a time interval size of $10^{-5} \mathrm{~s}$. This value is a good compromise since on

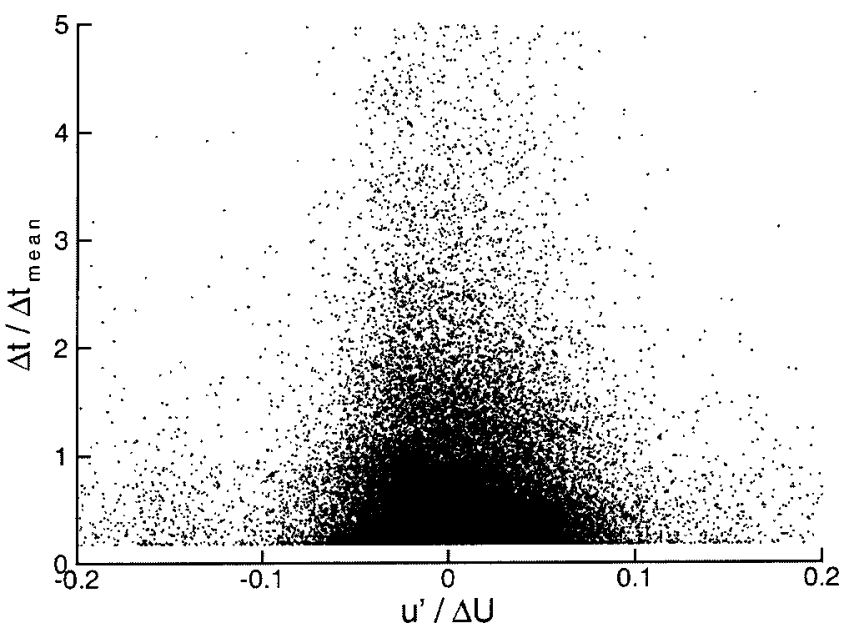

FIG. 26. Bursts quadrant view in an instantaneous velocity/time lag representation.

one hand it leads to an equivalent sampling frequency large enough for the studied phenomenon, and on the other hand each value of the correlation function is computed from more than 4000 samples.

In the resulting power spectrum, shown in Fig. 27, we observe a strong peak between 15.1 and $17.5 \mathrm{kHz}$. Assuming that this peak is due to the CJ intermittent penetration, the flapping frequency given by the LDV measurements is higher $(\approx 15 \%)$ than the frequency given by the visualizations in Sec. III B: $f_{\mathrm{CJ}}=13 \mathrm{kHz}$. However, visualization results are obtained by integrating in the streamwise direction, for $0.2<X / D<1.5$. In this region of the flow, the manipulated mixing layer thickness varies and is bigger than where LDV spectra analysis is made. The frequency obtained in Sec. III B corresponds to the structures in the CJ plume, which vary just like a standard local Kelvin-Helmholtz instability, rather than relative to the CJ flapping itself. Nevertheless, the CJ flapping frequency obtained by the LDV measurements is typically of the order of the nonmanipulated mixing layer frequency $f_{M L} \approx 15.3 \mathrm{kHz}$.

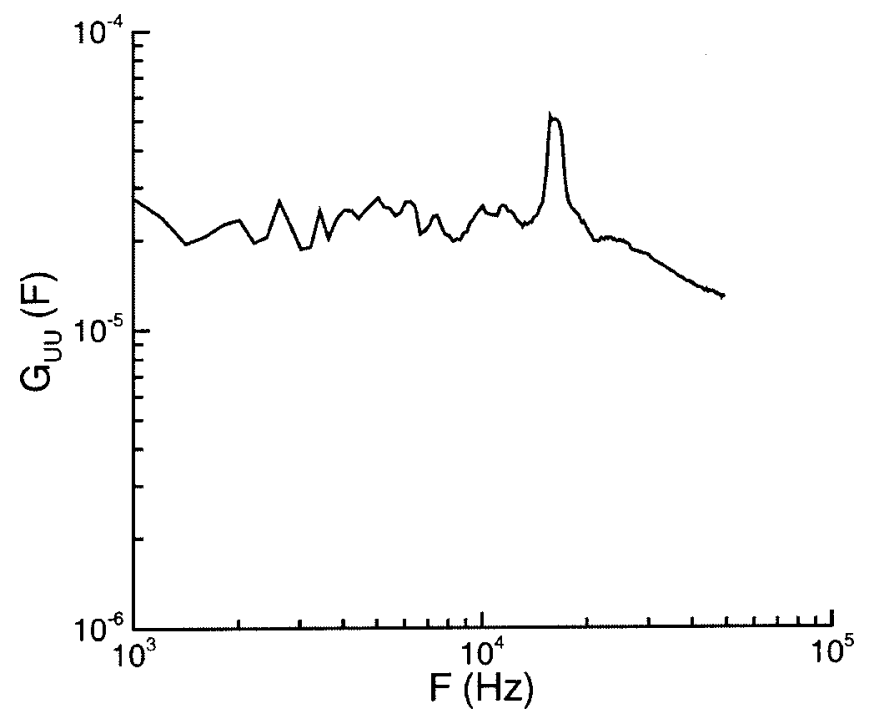

FIG. 27. $U$ component energy spectra at point B (cf. Fig. 15). 


\section{CONCLUSION}

A compressible single control jet (CJ)-mixing layer interaction has been studied experimentally in order to understand the mixing enhancement mechanism occurring in the very near field of this kind of flow. The main focus of the study was to accurately determine the nature of the interaction in terms of mean and turbulent flow quantities. Structural aspects of this flow were also studied. In particularly, the effect of this interaction on large scale turbulent structures was described and analyzed using instantaneous visualizations and LDV techniques.

The main results are:

The CJ is strongly intermittent and has a cyclic penetration into the mixing layer.

(ii) From instantaneous visualizations it seems that this CJ flapping occurs at a frequency very close to the natural local Kelvin-Helmholtz frequency of the initial mixing layer at the $\mathrm{CJ}$ location.

(iii) However, it seems that while the $\mathrm{KH}$ structures trigger this flapping motion, it cannot be attributed to the variation in apparent stagnation pressure (due to $\mathrm{KH}$ structure convection) of the mixing layer at the $\mathrm{CJ}$ location.

(iv) This cyclic effect may be explained by an intrinsic instability process in the near field of the interaction.

(v) The CJ-mixing layer interaction creates streamwise vortices but it is clear that the observed mixing enhancement seems linked to the penetration instability rather than to the longitudinal vorticity as originally assumed.

(vi) Mean velocity measurements show that a "return to isotropy" occurs rapidly and that the effects on the structure of the mean field do not persist downstream of $X / D \approx 0.3$, even if a strong mixing enhancement is present further downstream. The mixing layer thickness is increased locally but the downstream spreading rate quickly recovers its natural value.

(vii) All the turbulent intensities are increased in the interaction and this increase persists downstream of $X / D$ $\approx 0.6$, despite the mean velocity field having returned to an isotropy state at this location. The decrease in maximum shear stress after the interaction is exponential. With the exception of the anisotropy $\left(\sigma_{u} / \sigma_{v}\right.$, which seems to have a different evolution on the high speed side of the mixing layer), the spatial shape of the turbulent quantities are only slightly affected by the CJ interaction excepted for the anisotropy parameter. This effect can be attributed to a flapping shock created by the intermittent penetration of the $\mathrm{CJ}$ on the supersonic side of the mixing layer.

(viii) Quadrant and spectral analysis of the interaction near field clearly illustrate the dynamics of the CJ flapping motion. A measure of the characteristic frequency of this phenomenon was obtained by direct LDV measurements and confirms that it is close to the natural $\mathrm{KH}$ frequency of the undisturbed mixing layer at the CJ location.
It is clear that at the present time, measurements discussed here are somewhat restrictive: Since the CJ-mixing layer interaction involves very three-dimensional phenomena mainly in the very near field of the $\mathrm{CJ}$, further measurements are thus necessary. In particular, future measurements will be made to obtain the three velocity components $(u, v, w)$ at points in a three-dimensional mesh. However it appears that many particularities of the interaction can be explained by the present two-dimensional (2D) analysis.

Concerning the mixing performance aspect of this device, it seems that, although strong mixing is obtained immediately downstream of the CJ-mixing layer interaction, the effect is rapidly attenuated. Most notably, the mixing layer spreading rate recovers its natural value downstream of the interaction, showing that no strong structural change persists far downstream the $\mathrm{CJ}$, expectation of the degree of organization in the manipulated mixing layer.

\section{ACKNOWLEDGMENTS}

The authors would like to thank the companies LaVision and Spectra Physics for their technical support in the highspeed camera visualizations campaigns. Part of this work received the financial support from the DGA-SPAé.

${ }^{1}$ L. P. Bernal and A. Roshko, "Streamwise vortex structure in plane mixing layers," J. Fluid Mech. 170, 499 (1986).

${ }^{2}$ S. C. Crow and F. H. Champagne, "Orderly structure in jet turbulence," J. Fluid Mech. 48, 547 (1971)

${ }^{3}$ K. Yu, E. Gutmark, R. A. Smith, and K. C. Schadow, "Supersonic jet excitation using cavity-actuated forcing," AIAA Pap. 94-0185 (1994).

${ }^{4}$ G. Raman and E. J. Rice, "Supersonic jet mixing enhancement using impingement tones from obstacles of various geometries," AIAA J. 33, 454 (1995).

${ }^{5} \mathrm{U}$. Vandsburger and C. Ding, "Self excited wire method for the control of turbulent mixing layers," AIAA J. 33, 1032 (1995).

${ }^{6}$ N. D. Sandham and W. C. Reynolds, "Three-dimenional simulations of large eddies in the compressible mixing layer," J. Fluid Mech. 224, 133 (1991).

${ }^{7}$ L. J. Bradbury and A. H. Khadem, "The distortion of a jet by tabs," J. Fluid Mech. 70, 801 (1975).

${ }^{8}$ K. K. Ahuja and W. H. Brown, "Shear flow control by mechanical tabs," AIAA Pap. 89-0994 (1989).

${ }^{9}$ M. J. Carletti, C. B. Rogers, and D. E. Parekh, "Use of streamwise vorticity to increase mass entrainment in a cylindrical ejector," AIAA J. 33, 1641 (1995).

${ }^{10}$ F. F. Grinstein, E. J. Gutmark, T. P. Parr, D. M. Hanson-Parr, and U. Obeysekare, "Streamwise and spanwise vortex interaction in an axisymetric jet. A computational and experimental study," Phys. Fluids 8, 1515 (1996).

${ }^{11} \mathrm{~K}$. B. Zaman, "Axis switching and spreading of an asymetric jet: The role of coherent structure dynamics," J. Fluid Mech. 316, 1 (1996).

${ }^{12}$ J. Foss and K. B. Zaman, "Large- and small-scale vortical motions in a shear layer perturbated by tabs," J. Fluid Mech. 382, 307 (1999).

${ }^{13}$ J. H. Kim and M. Samimy, "Mixing enhancement via nozzle trailing edge modifications on a high speed rectangular jet," Phys. Fluids 11, 2731 (1999).

${ }^{14} \mathrm{G}$. Raman and E. J. Rice, "Axisymetric jet forced by fundamental and subharmonic tones," AIAA J. 29, 1114 (1991).

${ }^{15}$ H. E. Fielder and P. Mensing, "The plane turbulent shear layer with periodic excitation," J. Fluid Mech. 150, 281 (1985).

${ }^{16} \mathrm{~K}$. B. Zaman and G. Raman, "Reversal in spreading of a tabbed circular jet under controlled excitation," Phys. Fluids 9, 3733 (1997).

${ }^{17}$ N. T. Clemens and M. G. Mungal, "Two- and three-dimensional effects in the supersonic mixing layer," AIAA J. 30, 973 (1992).

${ }^{18} \mathrm{~N}$. T. Clemens and M. G. Mungal, "Large-scale structure and entrainment in the supersonic mixing layer," J. Fluid Mech. 294, 171 (1995).

${ }^{19}$ J. M. Wiltse and A. Glezer, "Manipulation of free shear flows using 
piezoelectric actuators," J. Fluid Mech. 249, 261 (1993).

${ }^{20}$ D. E. Parekh, V. Kibens, A. Glezer, J. M. Wiltse, and D. M. Smith, "Innovative jet flow control: Mixing enhancement experiments," AIAA Pap. 96-0308 (1996).

${ }^{21}$ D. Papamoschou, "Mixing enhancement using axial flow," AIAA Pap. 00-0093 (2000).

${ }^{22}$ K. B. Zaman and D. Papamoschou, "Study of mixing enhancement observed with a co-annular nozzle configuration," AIAA Pap. 00-0094 (2000).

${ }^{23}$ M. R. Davis, "Variable control of jet decay," AIAA J. 20, 606 (1982).

${ }^{24}$ J. B. Freund and P. Moin, "Jet mixing enhancement by high-amplitude fluidic actuatior," AIAA J. 38, 1863 (2000).

${ }^{25}$ J. Delville, E. Collin, S. Lardeau, S. Denis, E. Lamballais, S. Barre, and J. P. Bonnet, "Control of jets by radial fluid injection," ERCOFTAC Bulletin 44, 57 (2000).

${ }^{26}$ M. R. Lammari, "Mesures par vélocimétrie Laser Doppler dans une couche de mélange turbulente supersonique: quelques aspects du processus de mesure," Ph.D. thesis, University of Poitiers, France (1996).

${ }^{27}$ M. R. Lammari, P. Braud, S. Barre, and J. P. Bonnet, "Etude expérimentale du biais de vitesse en couche de mélange supersonique," $5^{\text {ème }}$ congrès francophone de vélocimétrie Laser, Rouen, France (1996)

${ }^{28} \mathrm{D}$. Alem, "Analyse expérimentale d'une turbulence homogéne en écoule- ment supersonique soumise à un choc droit," Ph.D. thesis, University of Poitiers, France (1995)

${ }^{29}$ S. Barre, D. Alem, and J. P. Bonnet, "Experimental study of a normal shock/homogeneous turbulence interaction," AIAA J. 34, 968 (1996).

${ }^{30}$ S. Bellaud, S. Barre, and J. P. Bonnet, "Experimental study of annular supersonic mixing layers: Turbulent kinetic energy budget," Turbulence and Shear Flow Phenomena-1, edited by S. Banerjee and J. K. Eaton (Begell, New York, 1999), p. 1103.

${ }^{31}$ S. Lardeau, E. Lamballais, and J. P. Bonnet, "Direct numerical simulation of a jet controlled by fluid injection," J. Turbulence 3, 002 (2002).

${ }^{32}$ E. Collin, S. Barre, and J. P. Bonnet, "Analysis of jet interaction for supersonic flow control," AVT-Symposium on Active Control Technology for Enhanced Performance Operation Capabilities of Military Aircraft, Land Verhicles and Sea Vehicles, Braunschweig, May 8-12, 2000, NATO-Applied Vehicle Technology Panel (AVT) (2000) [ftp:// ftp.rta.nato.int/pubfulltext/rto/mp/rto-mp-051/mp-051-psf-09.pdf].

${ }^{33}$ P. Huerre and P. A. Monkewitz, "Absolute and convective instabilities in free shear layers," J. Fluid Mech. 159, 151 (1985).

${ }^{34}$ M. Gaster and J. B. Roberts, "Spectral analysis of randomly sampled signals," J. Inst. Math. Appl. 15, 195 (1975).

${ }^{35}$ W. A. Bell, "Spectral analysis algorithms for the laser velocimeter: A comparative study," AIAA J. 21, 714 (1983). 